\title{
Simulation and Test on Vibration Characteristics of Power Transformer Windings
}

\author{
Li Hui1,a, Zhang Bin2,b,Chen Jiangbo1,c,Cao Chen2,d,Wang \\ Yang2,e \\ 1China Electric Power Research Institute, High Voltage Department, \\ Wuhan 430074, China \\ 2Shenyang University of Technology, Institute of Electrical Engineering, \\ Shenyang 110870, China \\ aemail: lihui2@epri.sgcc.com.cn, bemail:32652714@qq.com, cemail: \\ chenjiangbo@epri.sgcc.com.cn, demail: 406666428@qq.com,eemail: \\ 815411090@qq.com
}

\begin{abstract}
In order to researching the vibration characteristics of power transformer windings, the paper simulated the electromagnetic and structure coupling field of power transformer aimed at a type S11-M-500/35 power transformer and the 3D model of power transformer is built based on the method of multiple physics coupling. The voltage and current in secondary side, magnetic field distribution and vibration of power transformer is obtained under the condition of rated load current. The load test is made on the prototype to calibrate simulation model. Analysis results indicate that simulation results accords with experiment data well, which provides the evidence for researching the optimization and faults diagnosis of the power transformer.

Keywords: Power transformer; Vibration; Multiple physics coupling; Simulation; Test

\section{Introduction}

Power transformer is one of the most critical equipment in the power system whose operation reliability directly affect the operation of power grid security [1].The working characteristic of transformer has important effects on the operation of the power grid, Electrified winding under the action of magnetic field can generate electricity power, periodic oscillation of the electric power winding causes vibration. When the transformer short-circuit, winding vibration will not only affect the job performance of a transformer, and may even cause unbalanced ampere-turns and winding deformation phenomenon [2] [3] [4].Therefore, studying the characteristics of transformer winding vibration has the vital significance for the stable operation of the power grid.
\end{abstract}


Power transformer under the effect of electromagnetic excitation winding vibration problem of research, is actually a more physical field coupling calculation process. The Paper [5] power transformer body model is established based on finite element method, the transformer winding equivalent for mass spring system, winding short circuit conditions is studied in the axial vibration characteristics, but not to test the theory analysis and simulation results. Paper [6] adopts circuit - magnetic field coupling method, calculating electric power transformer running in different conditions, but there is no further research structure force field and electric field, magnetic field coupling solution.

This paper takes the S11-M-500kVA/35kV type transformer as the research object, Power transformer is established to coupled physics field of the 3D simulation model, coupling simulation electric field, magnetic field and structure field of the power transformer. Acquisition of transformer under rated loads, the relationship between the electrical parameters, the distribution of magnetic field and the vibration information. To carry out the vibration test under rated load transformer body, the test results verify the accuracy of transformer model.

\section{Mathematical model of vibration of transformer winding}

The winding leakage magnetic field generated around and winding under the effect of load current and leakage magnetic field produces electric power to cause the vibration of the winding, and pass on to other parts of the structure of transformer when the load current through the winding. What's more, when the transformer primary side or vice side short circuit, electromagnetic force for main transformer vibration exciting force [7].When the frequency characteristics of the winding and electromagnetism frequency close even overlap, producing a strong resonance. Every turn transformer coil by electromagnetic force is:

$$
F_{i}=i(t) B 2 \pi R
$$

In the formula, $\mathrm{Fi}$ is the coil by electromagnetic force, $\mathrm{i}(\mathrm{t})$ is a current in the coil, $\mathrm{B}$ is the location of the magnetic coil, $\mathrm{R}$ is the radius of the coil.

According to the structural characteristics of layer type distribution transformer winding, using insulating paper separated layer winding coil, with line type winding insulation between the block, compared to the elasticity of the insulation paper is far less than the insulation pad, so when the layer winding mechanics model is set up, the insulating paper between the coil can be equivalent to buffer, winding up and down at the ends of the insulation block can be equivalent to the elastic element. Winding mechanical model is shown in figure 1 . The coil use $\mathrm{m}$ instead of quality; Between the coil transformer oil with damping coefficient for C2 oil buffer instead; And the insulation of the coil end block with elastic coefficient of spring instead of K1, and K2. 


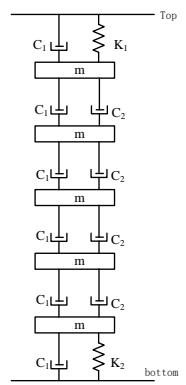

Fig.1. Transformer winding equivalent model of the system

Under the effect of electromagnetic force, the coil vibration dynamic mathematical model is:

$$
M \frac{d^{2} S}{d t^{2}}+C^{\prime} \frac{d S}{d t}+K^{\prime} S=F^{\prime}+M g
$$

$$
C^{\prime}=\sum_{i=1}^{n+1} C_{1}+\sum_{i=1}^{n-1} C_{2}
$$

$$
K^{\prime}=K_{1}+K_{2}
$$

$$
F^{\prime}=\sum_{i=1}^{n} F_{i}
$$

$$
M=\sum_{i=1}^{n} m
$$

In the formula, $\mathrm{n}$ is winding circle number; C' and $\mathrm{K}$ ', respectively is the total damping coefficient and stiffness coefficient of system; $S$ is winding the axial displacement of vibration; $\mathrm{M}$ and $\mathrm{F}$ ', respectively is winding total quality and general electric.

\section{Transformer vibration simulation analysis}

\section{Transformer field coupling modeling ideas more}

This topic research object is a S11 - M - 500/35 three-phase transformer. After considering the actual transformer iron core lamination section nearly round shape, As the transformer core of geometric model is set up, first of all, establish a geometric coordinate plane, establishing in $x-y$ plane core section of the graph, according to the actual core height, multiple profile graphics stretches along the $\mathrm{z}$ 
axis direction of circular core cylinder, Defined as the iron core of transformer core cylinder. Then according to the actual A/B/C three-phase high and low voltage coil size, in the outer core model of core column three groups of high and low voltage coil model is set up. We need to give material properties after geometric model is established. Giving the core model material properties of ferromagnetic materials, and input HB curve of ferromagnetic materials in the warehouse. Give the coil material properties of the model for the copper material, and the relative electric conductivity in the warehouse and electromagnetic parameters such as relative permeability. Main parameter of the coil in the magnetic field is defined. The main transformer coil parameters as shown in table 1.

Tab.1. Main parameters of the transformer coil

\begin{tabular}{ccc}
\hline Parameter & High voltage coil & Low voltage coils \\
\hline Number of turns & 2202 & 24 \\
Resistance (ohms) & 16.3 & 0.0014 \\
Coil type & Crossover coil & Layer type \\
\hline
\end{tabular}

This article in the solver settings in the options when solving three dimensional transient solver, in the solver configuration choose whole coupling solving method, and jacobian correction in each iteration. Sets the solving time is $0.05 \mathrm{~s}$, step is $0.001 \mathrm{~s}$, relative tolerance is 0.001 . Transformer coupled circuit, the structure of the magnetic field and force field of the model solving module meet the following differential equation:

$$
-\nabla \cdot \frac{\partial\left(\varepsilon_{0} \varepsilon_{r} \nabla V\right)}{\partial t}-\nabla \cdot\left(\sigma \nabla V-J^{e}\right)=0
$$

$$
\sigma \frac{\partial A}{\partial t}+\nabla \times\left(\mu_{0}^{-1} \mu_{r}^{-1} \nabla \times A\right)=J^{e}
$$

$$
m \frac{d^{2} u}{d t^{2}}+\xi \frac{d u}{d t}+k u=f(t)
$$

In the formula, $\varepsilon 0$ is a free space of dielectric constant,er is the relative dielectric constant, $\sigma$ is the electrical conductivity, Je is the external current density, $\mathrm{V}$ is a potential. $\mu 0$ is a free space magnetic permeability, $\mu \mathrm{r}$ is the relative magnetic permeability, $A$ is the vector magnetic potential, $m$ is a mass matrix, $\zeta$ is a damping coefficient matrix, $\mathrm{k}$ is stiffness coefficient matrix, $\mathrm{u}$ is the deformation displacement, $\mathrm{f}(\mathrm{t})$ is a stress.

Transformer winding more than physical field model is established and the simulation results

Before physical field coupling model established, first of all, set the peripheral 
drive circuit in the electric field in the module. In the high voltage coil of external circuit model applying frequency is $50 \mathrm{~Hz}$, the sinusoidal voltage source voltage amplitude is $1857 \mathrm{~V}$. In the low voltage winding open circuit model, low voltage at both ends of the coil nipple to simulate the load test in the secondary side short answer. Equivalent peripheral circuit model is shown in figure 2.

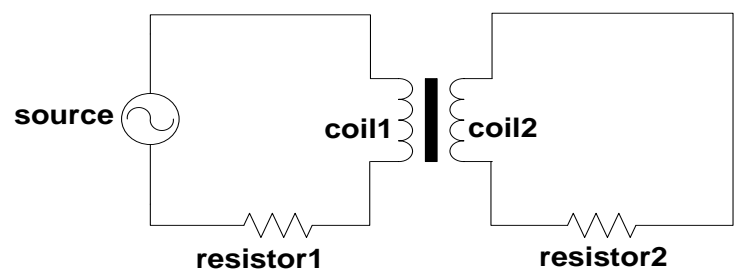

Fig.2. Transformer equivalent circuit model

By adding the ampere's law in a magnetic field model, linear vector magnetic potential, a scalar field domain boundary conditions such as the transformer body electromagnetic field model is established. Transformer coupling field model is established in this paper, need for grid subdivision model. After subdivision model as shown in figure 3.

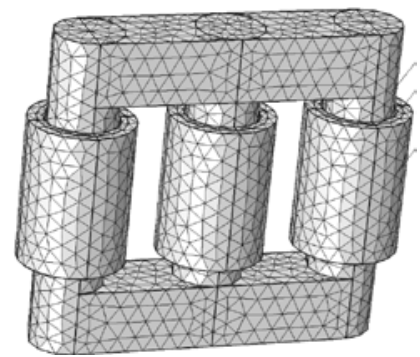

Fig.3. Transformer model as a whole

Transformer phase B is obtained by the simulation calculation of low-pressure coil induction coil current and B phase leakage magnetic flux density distribution as shown in figure 4 .

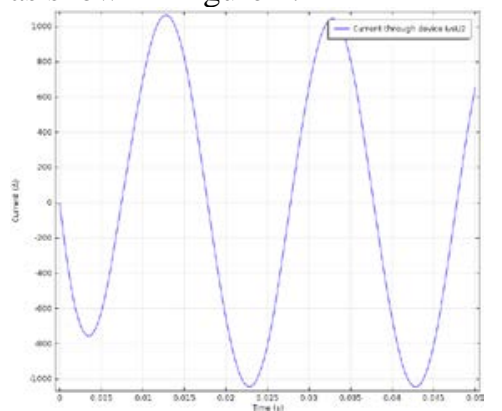

Low voltage B phase induction coil current

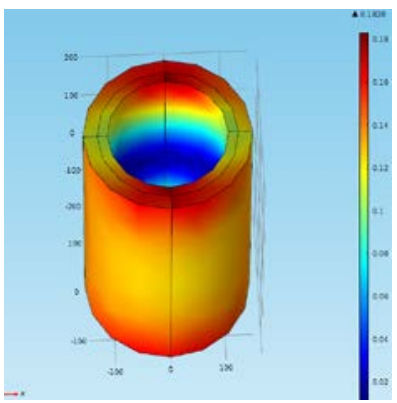

(b) B phase leakage magnetic flux density

Fig.4. Calculate low voltage transformer B phase induction coil and coil current leakage magnetic flux density distribution

As you can see from figure 4 and figure 5, low coil circuit module calculates 
the B phase induction current waveform amplitude for $1021 \mathrm{~A}$, frequency $50 \mathrm{~Hz}$ sine wave. Based on the results of simulation calculation, B in secondary side current effective value is $721.87 \mathrm{~A}$, the $\mathrm{B}$ phase leakage magnetic field distribution on the surface of the coil is analyzed, $n$ the coil of leakage magnetic field on both ends up and down flux density, maximum value for the maximum of $0.18 \mathrm{~T}$.

Transformer coil B phase is obtained by the simulation calculation of deformation displacement distribution as shown in figure 5 .

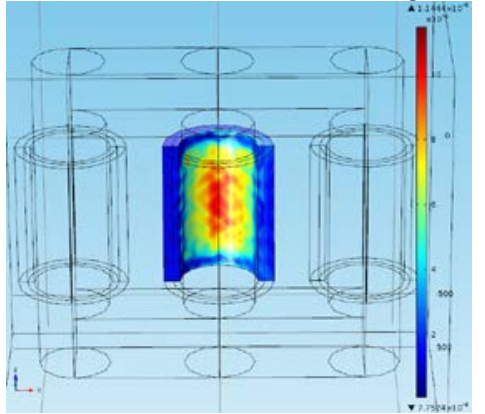

Fig.5. Deformation distribution transformer coil B phase

In figure 5, the greatest place in central coil winding deformation. This is because between the coil and iron core put insulating pad, after coil two ends up and down by insulating pad pressure tight, when the sinusoidal current cutting leakage magnetic field in the coil, at the end of the coil axial lorentz magnetic force. The lorentz force and pressure, advance coil will be from both ends to the middle axial function of extrusion, so the coil in the middle of the largest deformation trend.

\section{Test results}

In order to verify the accuracy of the model and the simulation results, the rated load test was carried out on the transformer body ontology, And in its current transformer (primary side current of $8.25 \mathrm{~A}$; secondary side current is 721.87 A) cases, measuring the vibration signals of the transformer winding. Vibration acquisition system using BK 3050 a vibration data acquisition front-end, sensor selection LC0154J voltage type acceleration sensor, sensitivity is $250 \mathrm{mV} / \mathrm{g}$, frequency range between $0.35 \mathrm{~Hz} \sim 3 \mathrm{kHz}$, range is $\pm 20 \mathrm{~g}$, three sensors using magnetic adsorption on large transformer body distance to $1 / 6$ position, The whole vibration acquisition system meet the testing requirements; Test principle and body field load test is shown in figure 6 . 

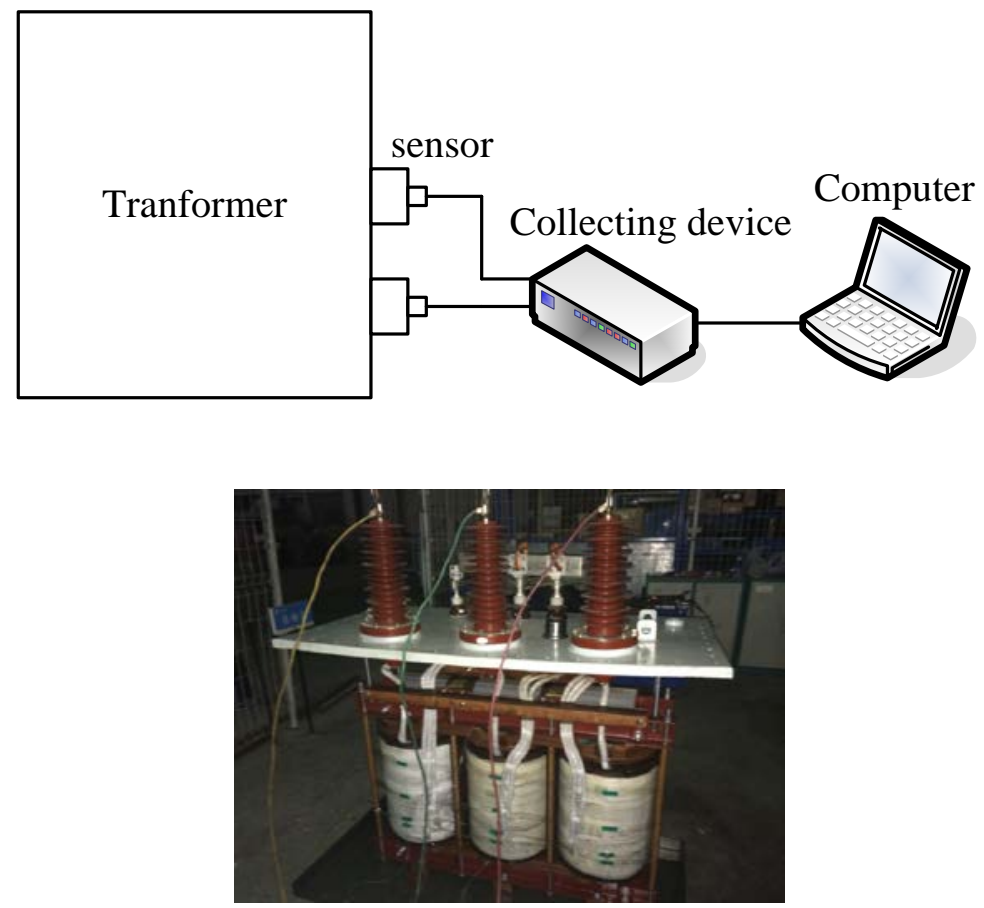

Fig.6. Test system structure and body load test site

According to the circuit, the structure of the magnetic field and force field coupling calculation results, time domain simulation model of the vibration signal can be transformer. Extraction of the vibration of the high voltage winding transformer model B phase 1/6 place simulation signal, and the large transformer body vibration signal measured in experiment were compared. Transformer model simulation and experiment of vibration measured B phase corresponding to the position of vibration signal contrast diagram as shown in figure 7.

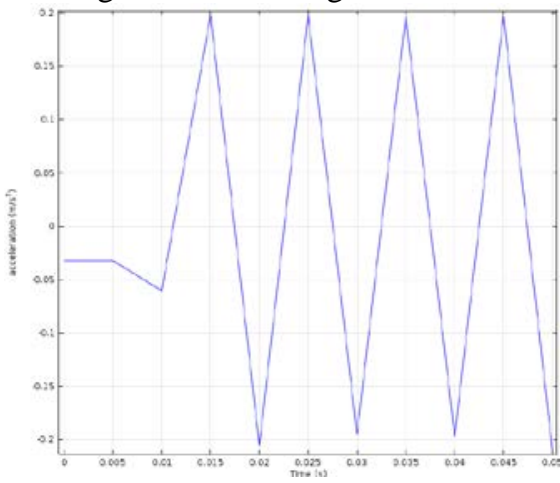




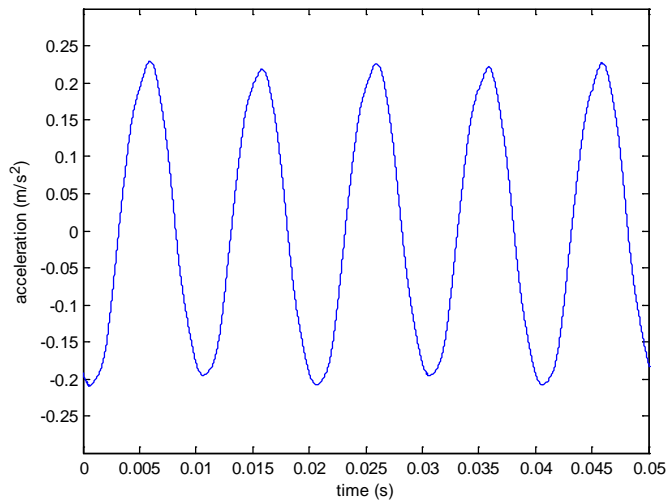

(a) B phase simulation of vibration signal vibration signal

(b) B phase measured of

Fig.7. Transformer simulation of vibration signal is compared with the actual vibration signal

Contrasting figure 7.(a) and figure 7.(b) shows that within 0.02s before simulation model in the simulation, the vibration amplitude smaller than normal. This is due to the selection of body in the structure field module load caused by inertial confinement. Simulation model of the maximum vibration amplitude value is $0.215 \mathrm{~m} / \mathrm{s} 2$, the maximum vibration amplitude of the test model is $0.23 \mathrm{~m} / \mathrm{s} 2$, simulation model and experimental prototype of the vibration signal frequency mainly is $100 \mathrm{~Hz}$. Simulation results are in good agreement with those obtained with the test data to validate the reliability of the simulation model, and proves that the accuracy of the theoretical research and simulation analysis.

\section{Conclusion}

In the paper, based on the physical field coupling method to establish a S11-M-500kVA/35kV type 3D simulation model of power transformer, power transformer electric field, magnetic field and structure field coupling simulation. Acquisition of transformer under rated loads, the relationship between the electrical parameters, the distribution of magnetic field and the vibration information. And rated load test was carried out on the transformer prototype, the simulation results and experimental data analysis, the following conclusions:

The transformer worked in rated load case, the simulation model of the secondary side current effective value is $721.87 \mathrm{~A}$, in conformity with the rated current of the transformer actual parameters. Circuit and the simulation results of the magnetic field can satisfy the requirements of transformer electromagnetic parameters. Simulation model of the maximum vibration amplitude value is $0.215 \mathrm{~m} / \mathrm{s} 2$, the maximum vibration amplitude of the test model is $0.23 \mathrm{~m} / \mathrm{s} 2$, the simulation results are in good agreement with experimental data. The accuracy of the simulation results are validated by test, in order to further study based on the 
vibration fault diagnosis of transformer winding and winding provide a basis for the optimization design.

\section{References}

[1] OU Xiao-bo, JI Sheng-chang, et al. Study on On-line Detecting of Transformer Winding Deformation Based on Parameter Identification of Leakage Reactance[J]. High Voltage Apparatus, 2010,46(12): 41-44.

[2] HAN Ai-zhi. Analysis of 220kV Transformer Fault and Its Treatment[J]. Transformer,2009,46(11): 71-73.

[3] Garcia B, Burgos J C, Alonso A M. Transformer tank vibration modeling as a method of detecting winding deformations-part II: experimental verification[J]. IEEE Transactions on Power Delivery,2006,21(1): 164-169.

[4] HU Wen-jia. Study of diagnosing method for transformer winding deformation[D].

[5] LI Yan, ZHOU Wei, JING Yong-teng, HAN Fang-xu. Axial Vibration Analysis on Active Part of Power Transformer under Short Circuit[J]. Adv. Tech. of Elec. Eng. \& Energy,2012,31(3): 49-53.

[6] WANG Shi-shah, LI Yan-ming. Computation of Electro dynamic Force on Windings of Transformer[J]. High Voltage Apparatus,2002,38(4): 22-25.

[7] Garcia B, Burgos J C, Alonso A M. Transformer tank vibration modeling as a method of detecting winding deformations-part I: theoretical foundation[J]. IEEE Transactions on Power Delivery,2006,21(1): 157-163. 